Mindestens die Hälfte des Buches ist dem verschlungenen geschichtlichen, geographischen, politischen, militärischen und wirtschaftlichen Umfeld der Meerengen und ihres Hinterlandes gewidmet (mit Seitenblicken auf die Antarktis und Falkland). Zum Verständnis der Rechtslage sind diese Informationen notwendig, denn es geht um die Verkehrs- und Zugangsrechte nicht nur für die Meerengen, sondern auch für die Fischerei und für wirkliche oder vermutete unterseeische Bodenschätze. Unverständlich bleibt, warum die Kartenskizzen gelegentlich unleserlich oder mißverständlich sind.

Der hier besonders interessierende völkerrechtliche Teil beginnt mit einer Darstellung des Grenzvertrages von 1881, der zugleich ein Meerengenvertrag über die Öffnung und Entmilitarisierung der Magellan-Straße war. Es folgten diverse bilaterale diplomatische Auseinandersetzungen, gelegentlich hart am Rande eines Krieges, die 1977 in einem Schiedsverfahren, einer nachfolgenen Streitschlichtung durch den Papst und schließlich 1984 in einem neuen Friedens- und Freundschaftsvertrag enden, der die Streitpunkte um Basislinien, Inseln, Wirtschaftszonen und Verkehrsrechte zwischen Chile und Argentinien endlich einvernehmlich regelt.

So liegt der Wert dieser Veröffentlichung für den politisch und völkerrechtlich Interessierten in der Darstellung eines wenig bekannten, aber äußerst brisanten Seerechtskonflikts, der unter Ausnutzung verschiedener Streitregelungsmechanismen letztlich zu einem guten Ergebnis führte, obwohl handfeste Wirtschaftsinteressen und das Prestige zweier lateinamerikanischer Nationen und ihrer Streitkräfte hier involviert waren.

Uwe Jenisch

\title{
United Nations Convention on the Law of the Sea 1982. A Commentary
}

Volume V, Settlement of Disputes, General and Final Provisions: Articles 279 to 320, Annexes V, VI, VII, VIII and IX, and Final Act, Annex 1, Resolutions I, II and IV Myron H. Nordquist, Editor-in-Chief

Martinus Nijhoff Publishers, Dordrecht / Boston / London 1989, 497 S., $£ 95.00$

Rechtzeitig zum Inkrafttreten des UN-Seerechts-Übereinkommens (im Dezember 1994) und zur bevorstehenden Errichtung des Internationalen Seegerichtshofs ISGH in Hamburg (Herbst 1996) sind in dem auf sechs Bände angelegten Seerechtskommentar des Center for Ocean Law and Policy, University of Virginia, die Bände I, II, und V erschienen; die übrigen Bände werden rasch folgen.

Der hier zu besprechende Band V behandelt die Streitregelung, also Artikel 279 bis 299 und die dazugehörigen Annexe sowie die sog. Allgemeinen Bestimmungen (Art 300 bis 304) und die Schlußbestimmungen (Art. 305 bis 320). Als verantwortliche Herausgeber dieses Bandes zeichnen Shabtai Rosenne, seinerzeit Leiter der israelischen Seerechtsdelegation, sowie Professor Louis B. Sohn, Harvard Law School. Beide gehören zu den Chef- 
architekten der seerechtlichen Streitregelung und zählen wie die Mitautoren Daniel Vignes vom EG-Ministerrat und Gritakumar E. Chitty vom Seerechtsbüro der Vereinten Nationen zu den besten Kennern der außerordentlich vielseitigen Streitregelung im neuen Seerecht.

In der Einführung wird die Entstehungsgeschichte der verschiedenen Streitregelungsinstrumente nachgezeichnet. Bereits 1973 im Meeresbodenausschuß, dem Vorläufer der 3. UN-Seerechtskonferenz, regten die USA ein umfassendes gerichtliches Streitregelungssystem mit der Ausweichmöglichkeit eines Schiedsverfahrens an, das dem endgültigen Verhandlungsergebnis von 1982 im Ergebnis bereits nahe kam.

Die Kommentierung - artikelweise - folgt stets demselben Aufbau. Nach Auflistung aller Konferenzmaterialien, einschließlich der inoffiziellen Dokumente zur Entstehungsgeschichte des jeweiligen Artikels, folgt die eigentliche Kommentierung in erklärenden Absätzen mit gelegentlich bis zu 40 Gliederungsziffern.

Der Inhalt der Kommentierung läßt gelegentlich Wünsche offen, denn es dominiert die akribische Entstehungsgeschichte, während die Fundstellen von einschlägigen Fachbüchern und Entscheidungssammlungen manchmal zu kurz kommen. Als Beispiel sei die Kommentierung des wichtigen Artikels 301 "peaceful uses of the seas" genannt. Obwohl sich hinter dieser Vorschrift die vielseitige Frage der Zulässigkeit (und der Grenzen) militärischer Nutzungen auf See in Krieg und Frieden verbirgt, erfährt der Leser auf zwei knappen Seiten relativ wenig zum Prinzip der friedlichen Nutzung. Ebenso hätte es sich bei der Kommentierung mancher Vorschriften zum Seegerichtshof angeboten, die vielfältigen Querverbindungen zum IGH und zu anderen internationalen Streitregelungsmechanismen einzubeziehen.

$\mathrm{Da} ß$ die Verfasser inhaltliche Kompromisse machen mußten, mag aus drei Gründen zu erklären sein. Die seerechtliche Streitregelung ist tatsächlich in vielerlei Hinsicht Neuland gegenüber älteren Systemen der Streitregelung. Es gibt bisher relativ wenige gute Monographien und fachliche Aufsätze im internationalen Schrifttum. Schließlich ist der schiere Umfang des Streitregelungssystems mit immerhin über 100 von 450 Artikeln des SRÜ und seiner Annexe zu bewältigen, was hier in lesbarer Weise auf 500 Seiten gelungen ist.

Nach der Lektüre bleibt die Erkenntnis, daß die Durchsetzung des Völkerrechts mit dem seerechtlichen Streitregelungssystem große Fortschritte gemacht hat. Erstmalig können natürliche und juristische Personen (Art. 187 SRÜ) ihre Rechte gegenüber Staaten und internationalen Organisationen (Meeresbodenbehörde) durchsetzen. Für den Meeresbergbau gibt es eine besondere Kammer mit umfangreichen obligatorischen Zuständigkeiten. Sie hat gegenüber der Meeresbodenbehörde auch gutachterliche, beratende Funktion. Für die Praxis der Schiffahrt sind Eilverfahren zur sofortigen Freilassung festgehaltener Schiffe besondes wichtig; sie wurden sogar von den notorischen Gegnern einer internationalen Streitbeilegung begrüßt. Die Vielfalt der Streitregelungsverfahren stellt natürlich einen Kompromiß zwischen den Befürwortern und Gegnern der internationalen Streitbeilegung dar, oder - um es positiv auszudrücken - das Streitregelungssystem ist benutzerfreundlich, indem es den Staaten Auswahlmöglichkeiten unter mehreren Streitbeilegungsverfahren gewährt, von denen zumindest das Vermittlungsverfahren verbindlich ist. 
Durch seine bloße Existenz wird dieses Streitregelungssystem, so ist zu hoffen, zum Frieden auf den Meeren beitragen, denn es schafft für die meisten Seerechtsstreitfälle Justitiabilität anstelle der bisher häufig üblichen (militärisch/politischen) Konfrontation. Ein internationales Gerichtsurteil wird in der Regel in der internationalen Praxis auf größere Akzeptanz stoßen als eine einseitige militärische Aktion.

Ein Schönheitsfehler mag sein, daß der Hamburger Seegerichtshof als obligatorische Urteilsinstanz nicht immer und automatisch zum Zuge kommen wird. Als attraktiv für die Praxis werden sich voraussichtlich die Schieds- und Vermittlungsverfahren erweisen, für die der ISGH sein Know-how und seine Richter im Einzelfall als Streitschlichter anbieten kann. So wird es für die Bedeutung des Seegerichtshofs entscheidend darauf ankommen, das Vertrauen der Vertragsparteien in seine Objektivität und Sachkunde zu gewinnen.

Der vorliegende Kommentar ist somit in seiner Art einzigartig und zum richtigen Zeitpunkt erschienen. Er wird für alle, die mit der Errichtung des Seegerichtshofs zu tun haben, für alle zukünftigen Nutzer der seerechtlichen Streitregelung sowie für Forschung und Lehre für längere Zeit unentbehrlich bleiben.

Uwe Jenisch

\section{Antonio Tanca}

\section{Foreign Armed Intervention in Internal Conflict}

Martinus Nijhoff Publishers, Dordrecht 1993, 243 pp., $£ 57.00$

Tanca's aim is to inquire into the system of norms regulating the "internationalization" of internal conflicts, his topic is ever important. Despite the fact that there is quite a lot of literature on this or related topics this one is especially well-researched and presented and certainly deserves special praise. Antonio Tanca has had the distinct advantage of having done his research and work on his doctoral thesis in Cambridge and Florence and carried out revision work at the Max-Planck-Institut in Heidelberg where he had acccess to excellent teachers and libraries.

"Through a careful examination of all relevant cases of 'internationalized' internal conflict from 1956, an attempt will be made to reassess the validity of the traditional framework of rules concerning foreign intervention in internal conflict. At the same time, the applicability to these situations of the rules typical of international conflicts will be analyzed with a view to proving the existence of continuum between the two situations, not only as a matter of fact but also with respect to their legal regulation." (p. 1)

In his introduction Tanca emphasizes the ban on use of force in the UN Charter, describes the 'grey areas' and elaborates on Art. 39. "(...) the Security Council has seldom acted as the 'protective arm' of the world community. Preservation of world peace has thus come to be entrusted to individual States by means of a rather rudimentary set of rules. The obvious 